\title{
A NOTE ON ASYMPTOTIC EXPONENTIAL ARBITRAGE WITH EXPONENTIALLY DECAYING FAILURE PROBABILITY
}

\author{
$\mathrm{KAI} \mathrm{DU}^{* * *}$ AND \\ ARIEL DAVID NEUFELD, ${ }^{* * * *}$ ETH Zürich
}

\begin{abstract}
The goal of this paper is to prove a result conjectured in Föllmer and Schachermayer (2007) in a slightly more general form. Suppose that $S$ is a continuous semimartingale and satisfies a large deviations estimate; this is a particular growth condition on the mean-variance tradeoff process of $S$. We show that $S$ then allows asymptotic exponential arbitrage with exponentially decaying failure probability, which is a strong and quantitative form of long-term arbitrage. In contrast to Föllmer and Schachermayer (2007), our result does not assume that $S$ is a diffusion, nor does it need any ergodicity assumption.
\end{abstract}

Keywords: Asymptotic exponential arbitrage; continuous semimartingale model; large deviations

2010 Mathematics Subject Classification: Primary 91G10

Secondary 60F10; 60G44

\section{Introduction}

Let $(\Omega, \mathcal{F}, \mathbb{F}, \mathrm{P})$ be a filtered probability space where the filtration $\mathbb{F}=\left(\widetilde{F}_{t}\right)_{t \geq 0}$ satisfies the usual conditions, and let the price process $S=\left(S_{t}\right)_{t \geq 0}$ initially be any $\mathbb{R}^{d}$-valued semimartingale. We define, for each $T>0$, the set

$$
\mathbb{K}^{T}:=\left\{\int_{0}^{T} H_{S} \mathrm{~d} S_{S} \mid H \in L(S) \text { admissible, i.e. } \int H \mathrm{~d} S \geq-a \text { for some } a \in \mathbb{R}_{+}\right\} .
$$

The following form of a long-term arbitrage was considered for the first time in [1]; its name is taken from [4].

Definition 1.1. The process $S=\left(S_{t}\right)_{t \geq 0}$ allows asymptotic exponential arbitrage with exponentially decaying failure probability if there exist $0<\tilde{T}<\infty$ and constants $C, \gamma_{1}, \gamma_{2}>0$ such that, for all $T \geq \tilde{T}$, there is $X_{T} \in \mathbb{K}^{T}$ with

(a) $X_{T} \geq-\mathrm{e}^{-\gamma_{1} T}$, P-almost surely (P-a.s.),

(b) $\mathrm{P}\left[X_{T} \leq \mathrm{e}^{\gamma_{1} T}\right] \leq C \mathrm{e}^{-\gamma_{2} T}$.

If $S$ has that property, we can find, for any large enough maturity $T$, up to an exponentially (in $T$ ) small probability of failure, an exponentially (in $T$ ) large profit with an exponentially

Received 24 July 2012; revision received 9 January 2013.

* Postal address: Department of Mathematics, ETH Zürich, 8092 Zürich, Switzerland.

** Email address: kai.du@math.ethz.ch

*** Email address: ariel.neufeld@math.ethz.ch 
(in $T$ ) small potential loss. This gives an explicit relation between any tolerance level of failure and the necessary time to reach a high level. Furthermore, when $T \rightarrow \infty$, we get in the limit a riskless profit. Thus, asymptotic exponential arbitrage with exponentially decaying failure probability can be interpreted as a strong and quantitative form of long-term arbitrage.

We define the sets

$\mathbb{M}_{m}^{T, e}:=\left\{\mathrm{Q}\right.$ probability measure on $\mathcal{F}_{T}|\mathrm{Q} \approx \mathrm{P}|_{\mathcal{F}_{T}}$ and $\left(S_{t}\right)_{0 \leq t \leq T}$ is a local Q-martingale $\}$.

Assumption 1.1. Throughout this paper, we assume that $\mathbb{M}_{m}^{T, e} \neq \varnothing$ for any $0<T<\infty$ and that the filtration $\mathbb{F}$ is continuous, i.e. every local martingale with respect to $\mathbb{F}$ is continuous.

We show below that, under Assumption 1.1, any semimartingale in $\mathbb{F}$ is in fact continuous. Moreover, using a result of Schweizer [5], we show in Lemma 2.2 that there exists a predictable, sufficiently integrable $\mathbb{R}^{d}$-valued process $\lambda=\left(\lambda_{t}\right)_{t \geq 0}$ such that, for any $T<\infty$ and any $\mathrm{Q} \in \mathbb{M}_{m}^{T, e}$, the density process $Z^{\mathrm{Q}}=\left(Z_{t}^{\mathrm{Q}}\right)_{0 \leq t \leq T}$ of $\mathrm{Q}$ with respect to $\left.\mathrm{P}\right|_{\mathcal{F}_{T}}$ is of the form

$$
Z^{\mathrm{Q}}=Z_{0}^{\mathrm{Q}} \mathcal{E}\left(\int-\lambda \mathrm{d} M+N^{\mathrm{Q}}\right)=: Z_{0}^{\mathrm{Q}} \mathcal{E}\left(L^{\mathrm{Q}}\right) \text { on } \llbracket 0, T \rrbracket,
$$

where $N^{\mathrm{Q}}=\left(N_{t}^{\mathrm{Q}}\right)_{0 \leq t \leq T}$ is a continuous local martingale with $N^{\mathrm{Q}} \perp M^{T}$ and $M^{T}$ is the continuous local martingale coming from the canonical decomposition of $S^{T}$. We call $\lambda$ a market price of risk for the price process $S$.

Following Föllmer and Schachermayer [1], we extend the notion of $S$ satisfying a large deviations estimate.

Definition 1.2. A market price of risk $\lambda=\left(\lambda_{t}\right)_{t \geq 0}$ for the price process $S=\left(S_{t}\right)_{t \geq 0}$ satisfies a large deviations estimate if there exist constants $c_{1}, c_{2}>0$ such that

$$
\limsup _{T \rightarrow \infty} \frac{1}{T} \log \mathrm{P}\left[\frac{1}{T} \int_{0}^{T} \lambda_{s}^{\operatorname{tr}} \mathrm{d}\langle M\rangle_{s} \lambda_{s} \leq c_{1}\right]<-c_{2} .
$$

The main goal of this paper is to prove that, under Assumption 1.1, if a market price of risk for the price process $S$ satisfies a large deviations estimate, then $S$ allows asymptotic exponential arbitrage with exponentially decaying failure probability.

In Föllmer and Schachermayer [1], the authors considered an $\mathbb{R}^{d}$-valued diffusion $\tilde{S}=$ $\left(\tilde{S}_{t}\right)_{t \geq 0}$ defined over a filtered probability space $(\tilde{\Omega}, \tilde{\mathcal{F}}, \tilde{\mathbb{F}}, \tilde{\mathrm{P}})$, where the filtration $\tilde{\mathbb{F}}=\left(\tilde{\mathcal{F}}_{t}\right)_{t \geq 0}$ is the $\tilde{\mathrm{P}}$-augmentation of the raw filtration generated by an $\mathbb{R}^{N}$-valued Brownian motion $\tilde{W}$ and $\tilde{S}$ is of the form

$$
\mathrm{d} \tilde{S}_{t}=\sigma\left(\tilde{S}_{t}\right)\left(\mathrm{d} \tilde{W}_{t}+\varphi\left(\tilde{S}_{t}\right) \mathrm{d} t\right) .
$$

In (1.2), $\sigma: \mathbb{R}^{d} \rightarrow \mathbb{R}^{d \times N}$ and $\varphi: \mathbb{R}^{d} \rightarrow \mathbb{R}^{N}$ are such that $\varphi\left(\tilde{S}_{t}\right) \in\left(\operatorname{ker}\left(\sigma\left(\tilde{S}_{t}\right)\right)^{\perp}\right.$ for any $t \geq 0$ and the process $\tilde{Z}=\left(\tilde{Z}_{t}\right)_{t \geq 0}$ defined by

$$
\tilde{Z}_{t}:=\mathcal{E}\left(-\int \varphi(\tilde{S}) \mathrm{d} \tilde{W}\right)_{t}=\exp \left(-\int_{0}^{t} \varphi\left(\tilde{S}_{s}\right) \mathrm{d} \tilde{W}_{s}-\frac{1}{2} \int_{0}^{t}\left\|\varphi\left(\tilde{S}_{s}\right)\right\|^{2} \mathrm{~d} s\right)
$$

is a strictly positive $\tilde{\mathrm{P}}$-martingale, where $\|\cdot\|$ denotes the Euclidean norm on $\mathbb{R}^{N}$.

Definition 1.3. The market price of risk function $\varphi(\cdot)$ for the price process $\tilde{S}$ satisfies a large deviations estimate with respect to $\tilde{S}$ if there are constants $c_{1}, c_{2}>0$ such that

$$
\limsup _{T \rightarrow \infty} \frac{1}{T} \log \mathrm{P}\left[\frac{1}{T} \int_{0}^{T}\left\|\varphi\left(\tilde{S}_{S}\right)\right\|^{2} \mathrm{~d} s \leq c_{1}\right]<-c_{2} .
$$


Föllmer and Schachermayer [1] formulated the conjecture that if (1.4) holds, then $\tilde{S}$ allows asymptotic exponential arbitrage with exponentially decaying failure probability. In Mbele Bidima and Rásonyi [4], the authors proved such a result in a discrete-time version of the model (1.2) with bounded drift and volatility. In the present paper we can show, as a corollary of our main theorem, that the conjecture is also true in the stated form for the continuous-time price process $\tilde{S}$ in (1.2).

\section{Main theorem, its proof, and comments}

We begin by showing the following result.

Lemma 2.1. Under Assumption 1.1, the process $S$ is continuous.

Proof. This is well known, but we present a proof for completeness. Take any decomposition $S=S_{0}+M+A$ with a local martingale $M$ and with $A$ of finite variation. Take any $T<\infty$ and any $\mathrm{Q} \in \mathbb{M}_{m}^{T, e}$ with density process $Z=\left(Z_{t}\right)_{0 \leq t \leq T}$ with respect to $\left.\mathrm{P}\right|_{\mathcal{F}_{T}}$. As $\mathbb{F}$ is continuous, the local P-martingales $Z, Z S$, and $M$, and, hence, also $1 / Z$, are continuous processes up to time $T$. So $A=(1 / Z) Z S-M-S_{0}$ and, hence, also $S$ are continuous up to time $T$, which gives the result.

Notation. Under Assumption 1.1, we let

$$
S=S_{0}+M+A
$$

be the canonical decomposition of the continuous semimartingale $S$, where $M$ is a continuous local martingale and $A$ is a continuous process of finite variation.

We next characterize for any $\mathrm{Q} \in \mathbb{M}_{m}^{T, e}$ the structure of its density process $Z^{\mathrm{Q}}$ with respect to $\left.\mathrm{P}\right|_{\mathcal{F}_{T}}$. For unexplained notation from martingale theory, we refer the reader to [2].

Lemma 2.2. Under Assumption 1.1, there exists an $\mathbb{R}^{d}$-valued process $\lambda=\left(\lambda_{t}\right)_{t \geq 0} \in L_{\text {loc }}^{2}(M)$ such that, for any $T<\infty$ and any $\mathrm{Q} \in \mathbb{M}_{m}^{T, e}$, the density process $Z^{\mathrm{Q}}=\left(Z_{t}^{\mathrm{Q}}\right)_{0 \leq t \leq T}$ of $\mathrm{Q}$ with respect to $\left.\mathrm{P}\right|_{\mathcal{F}_{T}}$ is of the form

$$
Z^{\mathrm{Q}}=Z_{0}^{\mathrm{Q}} \mathcal{E}\left(\int-\lambda \mathrm{d} M+N^{\mathrm{Q}}\right)=: Z_{0}^{\mathrm{Q}} \mathcal{E}\left(L^{\mathrm{Q}}\right) \text { on } \llbracket 0, T \rrbracket,
$$

where $N^{\mathrm{Q}}=\left(N_{t}^{\mathrm{Q}}\right)_{0 \leq t \leq T}$ is a continuous local martingale with $N^{\mathrm{Q}} \perp M^{T}$. As a consequence, we have

$$
\left\langle L^{\mathrm{Q}}\right\rangle_{t} \geq \int_{0}^{t} \lambda_{s}^{\operatorname{tr}} \mathrm{d}\langle M\rangle_{s} \lambda_{s}
$$

for each $t \in[0, T]$. We call $\lambda$ a market price of risk for the price process $S$.

Proof. By Lemma 2.1, the process $S$ is continuous. Since $\mathbb{M}_{m}^{T, e} \neq \varnothing$ for any $T<\infty$, Theorem 1 of [5] gives, for any $T<\infty$, an $\mathbb{R}^{d}$-valued process $\lambda^{(T)}=\left(\lambda_{t}^{(T)}\right)_{0 \leq t \leq T} \in L_{\text {loc }}^{2}\left(M^{T}\right)$ such that, for any $\mathrm{Q} \in \mathbb{M}_{m}^{T, e}$, the density process $Z^{\mathrm{Q}}=\left(Z_{t}^{\mathrm{Q}}\right)_{0 \leq t \leq T}$ of $\mathrm{Q}$ with respect to $\left.\mathrm{P}\right|_{\mathcal{F}_{T}}$ is of the form

$$
Z^{\mathrm{Q}}=Z_{0}^{\mathrm{Q}} \mathcal{E}\left(\int-\lambda^{(T)} \mathrm{d} M^{T}+N^{\mathrm{Q}}\right) \quad \text { on } \llbracket 0, T \rrbracket,
$$

where $N^{\mathrm{Q}}=\left(N_{t}^{\mathrm{Q}}\right)_{0 \leq t \leq T}$ is a continuous local martingale with $N^{\mathrm{Q}} \perp M^{T}$. We point out that the process $\lambda^{(T)}$ need not be unique. However, the stochastic integral $\int \lambda^{(T)} \mathrm{d} M^{T}$ does not depend 
on the choice of $\lambda^{(T)}$ satisfying (2.3); see [5]. Extending the process $\lambda^{(T)}$ to $[0, \infty)$ by setting $\bar{\lambda}^{(T)}=\lambda^{(T)} \mathbf{1}_{\llbracket 0, T \rrbracket}$, we clearly have $\bar{\lambda}^{(T)} \in L_{\text {loc }}^{2}(M)$. The $\mathbb{R}^{d}$-valued process $\lambda=\left(\lambda_{t}\right)_{t \geq 0}$ defined by

$$
\lambda:=\sum_{n=1}^{\infty} \bar{\lambda}^{(n)} \mathbf{1}_{((n-1, n \rrbracket}
$$

is then in $L_{\text {loc }}^{2}(M)$, too. Moreover, $\left.\mathrm{Q}\right|_{\mathcal{F}_{n-1}} \in \mathbb{M}_{m}^{n-1, e}$ for any $\mathrm{Q} \in \mathbb{M}_{m}^{n, e}$, and so (2.3) inductively yields

$$
\int \lambda \mathrm{d} M=\int \bar{\lambda}^{(n)} \mathrm{d} M=\int \bar{\lambda}^{(n)} \mathrm{d} M^{n} \quad \text { on } \llbracket 0, n \rrbracket
$$

for any $n \in \mathbb{N}$. So (2.1) follows from (2.3) and (2.4).

Finally, $L^{\mathrm{Q}}=-\int \lambda \mathrm{d} M+N^{\mathrm{Q}}$ on $\llbracket 0, T \rrbracket$ and $N^{\mathrm{Q}} \perp M^{T}$ imply (2.2) because

$$
\left\langle L^{\mathrm{Q}}\right\rangle=\int \lambda^{\operatorname{tr}} \mathrm{d}\langle M\rangle \lambda+\left\langle N^{\mathrm{Q}}\right\rangle .
$$

Remark 2.1. We do not claim that the market price of risk $\lambda$ for the price process $S$ is unique. However, as already used, the stochastic integral $\int \lambda \mathrm{d} M$ does not depend on the choice of $\lambda$. This can, for instance, be seen by writing for $\mathrm{Q} \in \mathbb{M}_{m}^{T, e}$ the density process $Z^{\mathrm{Q}}=Z_{0}^{\mathrm{Q}} \mathcal{E}\left(L^{\mathrm{Q}}\right)$ and then arguing that $-\int \lambda \mathrm{d} M$ must be the projection of $L^{\mathrm{Q}}$ on $M$; this follows because $Z^{\mathrm{Q}} S$ is a local P-martingale. As a consequence, the property of satisfying a large deviations estimate does not depend on the choice of the market price of risk $\lambda$ either.

Notation. For brevity, we introduce the so-called mean-variance tradeoff process

$$
K_{t}:=\int_{0}^{t} \lambda_{s}^{\operatorname{tr}} \mathrm{d}\langle M\rangle_{s} \lambda_{s}
$$

for $t \geq 0$. This process is finite valued since $\lambda \in L_{\text {loc }}^{2}(M)$, and it does not depend on the choice of the market price of risk $\lambda$; in fact, $K=\left\langle\int \lambda \mathrm{d} M\right\rangle$.

Lemma 2.3. Under Assumption 1.1, suppose that a market price of risk $\lambda$ for the price process $S$ satisfies a large deviations estimate. Then

$$
K_{\infty}:=\lim _{t \rightarrow \infty} K_{t}=\infty \quad \text { P-a.s. }
$$

Proof. If the above statement is not true, there is a constant $C>0$ with

$$
\mathrm{P}\left[K_{\infty} \leq C\right]=: \mathrm{P}[B]>0 .
$$

As $\lambda$ satisfies a large deviations estimate, there exist constants $c_{1}, c_{2}>0$ such that

$$
\limsup _{T \rightarrow \infty} \frac{1}{T} \log \mathrm{P}\left[\frac{1}{T} K_{T} \leq c_{1}\right]=: \limsup _{T \rightarrow \infty} \frac{1}{T} \log \mathrm{P}\left[V_{T}\right]<-c_{2} .
$$

Thus, we can find $0<\bar{T}<\infty$ such that

$$
C \leq c_{1} \bar{T}, \quad \mathrm{P}\left[V_{\bar{T}}\right] \leq \mathrm{e}^{-c_{2} \bar{T} / 2}, \quad \mathrm{e}^{-c_{2} \bar{T} / 2}<\mathrm{P}[B] .
$$

As $C \leq c_{1} \bar{T}$ and $K$ is increasing, we get $B \subseteq V_{\bar{T}}$. But then, by the definition of $\bar{T}$,

$$
\mathrm{P}[B] \leq \mathrm{P}\left[V_{\bar{T}}\right] \leq \mathrm{e}^{-c_{2} \bar{T} / 2}<\mathrm{P}[B],
$$

which gives a contradiction. 
Lemma 2.4. Under Assumption 1.1, suppose that a market price of risk $\lambda$ for the price process $S$ satisfies a large deviations estimate. Fix $0<T<\infty$, and let $L=\left(L_{t}\right)_{0 \leq t \leq T}$ be a continuous local martingale with $L_{0}=0$. Then there exists a continuous local martingale $\bar{L}=\left(\bar{L}_{t}\right)_{t \geq 0}$ defined for all times $t \in[0, \infty)$ such that $\bar{L}_{t}=L_{t}$ for any $t \in[0, T]$ and

$$
\langle\bar{L}\rangle_{\infty}:=\lim _{t \rightarrow \infty}\langle\bar{L}\rangle_{t}=\infty \quad \text { P-a.s. }
$$

Proof. Define the process $Y=\left(Y_{t}\right)_{t \geq T}$ by $Y_{t}:=\int_{T}^{t} \lambda_{s} \mathrm{~d} M_{s}$, set $\bar{Y}:=Y \mathbf{1}_{\llbracket T, \infty))}$ and

$$
\bar{L}:=L \mathbf{1}_{\llbracket 0, T \rrbracket}+\bar{Y}=L \mathbf{1}_{\llbracket 0, T \rrbracket}+Y \mathbf{1}_{\llbracket T, \infty))} .
$$

Then $\bar{L}$ is a continuous local martingale null at 0 like $L, Y$, and $\bar{Y}$, and we have $\bar{L}=L$ on $\llbracket 0, T \rrbracket$ by construction. Moreover,

$$
\langle\bar{L}\rangle_{\infty}=\langle L\rangle_{T}+\langle\bar{Y}\rangle_{\infty}=\langle L\rangle_{T}+\int_{T}^{\infty} \lambda_{s}^{\operatorname{tr}} \mathrm{d}\langle M\rangle_{s} \lambda_{s}=\langle L\rangle_{T}+K_{\infty}-K_{T}=\infty \quad \text { P-a.s. }
$$

due to Lemma 2.3.

Remark 2.2. In Lemma 2.4, we can replace Assumption 1.1 and the condition on $\lambda$ by assuming instead that there exists a Brownian motion $B$ with respect to the filtration $\mathbb{F}$, which is a much weaker assumption. Indeed, in that case, we just define in the above proof the process $Y$ by $Y_{t}:=B_{t}-B_{T}$ for $t \geq T$. The rest of the argument then works in the same way.

Following Föllmer and Schachermayer [1], we now define the notion of $\left(\varepsilon_{1}, \varepsilon_{2}\right)$-arbitrage (up to time $T$ ).

Definition 2.1. Fix any $T<\infty$, and let $0<\varepsilon_{1}, \varepsilon_{2}<1$. The process $S$ admits an $\left(\varepsilon_{1}, \varepsilon_{2}\right)$ arbitrage up to time $T$ if there exists $X_{T} \in \mathbb{K}^{T}$ such that

(a) $X_{T} \geq-\varepsilon_{2}$, P-a.s.,

(b) $\mathrm{P}\left[X_{T} \geq 1-\varepsilon_{2}\right] \geq 1-\varepsilon_{1}$.

Our next preliminary result is a direct consequence of Proposition 2.3 of [1]. More precisely, the result follows by the implications (ii) $\Rightarrow$ (iii) $\Rightarrow$ (i) in that proposition. See also Remark 2.4 of [1].

Lemma 2.5. Fix any $T<\infty$, and let $0<\varepsilon_{1}, \varepsilon_{2}<1$ be such that, for each $\mathrm{Q} \in \mathbb{M}_{m}^{T, e}$, there is a set $A_{T}^{\mathrm{Q}} \in \mathcal{F}_{T}$ with $\mathrm{P}\left[A_{T}^{\mathrm{Q}}\right] \leq \varepsilon_{1}$ and $\mathrm{Q}\left[A_{T}^{\mathrm{Q}}\right] \geq 1-\varepsilon_{2}$. Then, for any $0<\tilde{\varepsilon}_{1}, \tilde{\varepsilon}_{2}<1$ with $2^{1+\alpha} \max \left(\varepsilon_{1}, \varepsilon_{2}^{\alpha}\right) \leq \tilde{\varepsilon}_{1} \tilde{\varepsilon}_{2}^{\alpha}$ for some $0<\alpha<\infty$, S admits an $\left(\tilde{\varepsilon}_{1}, \tilde{\varepsilon}_{2}\right)$-arbitrage up to time $T$.

Note that $\varepsilon_{1}$ and $\varepsilon_{2}$ in the assumption of Lemma 2.5 are exogenously given and unrelated to $T$. The point of the next result is that it allows us to choose them both exponentially small in $T$, if $S$ satisfies the extra condition of a large deviations estimate. This is the key for subsequently proving our main result.

Proposition 2.1. Under Assumption 1.1, suppose that a market price of risk $\lambda$ for the price process $S$ satisfies a large deviations estimate. Then there exist constants $\tilde{C}, \gamma_{1}, \gamma_{2}>0$ and $1 \leq T_{0}<\infty$ such that, for all $T \geq T_{0}$, we can find, for any $\mathrm{Q} \in \mathbb{M}_{m}^{T, e}$, a set $A_{T}^{\mathrm{Q}} \in \mathcal{F}_{T}$ with

$$
\mathrm{P}\left[A_{T}^{\mathrm{Q}}\right] \leq \tilde{C} \mathrm{e}^{-\gamma_{1} T}<1 \quad \text { and } \quad \mathrm{Q}\left[A_{T}^{\mathrm{Q}}\right] \geq 1-\mathrm{e}^{-\gamma_{2} T} .
$$


Proof. By assumption, there exist constants $c_{1}, c_{2}>0$ such that, as in (1.1),

$$
\limsup _{T \rightarrow \infty} \frac{1}{T} \log \mathrm{P}\left[\frac{1}{T} K_{T} \leq c_{1}\right]<-c_{2} .
$$

We take any constant $0<\delta<c_{1} / 2$ and set

$$
\gamma_{1}:=\min \left\{\frac{\left(c_{1}-2 \delta\right)^{2}}{8 c_{1}}, \frac{c_{2}}{2}\right\}>0, \quad \gamma_{2}:=\delta>0, \quad \tilde{C}:=\frac{\sqrt{2 c_{1}}}{\left(c_{1}-2 \delta\right) \sqrt{\pi}}+1>0 .
$$

By the definition of $\lim \sup _{T \rightarrow \infty}$, we find $1 \leq T_{0}<\infty$ such that, for all $T \geq T_{0}$,

$$
\tilde{C} \mathrm{e}^{-\gamma_{1} T}<1 \text { and } \mathrm{P}\left[K_{T} \leq c_{1} T\right] \leq \mathrm{e}^{-c_{2} T / 2} .
$$

Fix $T \geq T_{0}$ and $\mathrm{Q} \in \mathbb{M}_{m}^{T, e}$. For any stopping time $\sigma \leq T$, Lemma 2.2 gives

$$
\left.\frac{\mathrm{dQ}}{\mathrm{dP}}\right|_{\mathcal{F}_{\sigma}}=Z_{\sigma}^{\mathrm{Q}}=\exp \left(L_{\sigma}^{\mathrm{Q}}-\frac{1}{2}\left\langle L^{\mathrm{Q}}\right\rangle_{\sigma}\right) \text { with }\left\langle L^{\mathrm{Q}}\right\rangle_{\sigma} \geq K_{\sigma} .
$$

We define the set $G_{T}^{\mathrm{Q}}:=\left\{\left\langle L^{\mathrm{Q}}\right\rangle_{T}>c_{1} T\right\}$. Then (2.6) and (2.7) imply that

$$
\mathrm{P}\left[\left(G_{T}^{\mathrm{Q}}\right)^{c}\right]=\mathrm{P}\left[\left\langle L^{\mathrm{Q}}\right\rangle_{T} \leq c_{1} T\right] \leq \mathrm{P}\left[K_{T} \leq c_{1} T\right] \leq \mathrm{e}^{-c_{2} T / 2} .
$$

Now, Lemma 2.4 yields a continuous local martingale $\bar{L}^{\mathrm{Q}}=\left(\bar{L}_{t}^{\mathrm{Q}}\right)_{t \geq 0}$ with $\bar{L}^{\mathrm{Q}}=L^{\mathrm{Q}}$ on $\llbracket 0, T \rrbracket$ and $\left\langle\bar{L}^{\mathrm{Q}}\right\rangle_{\infty}=\infty$, P-a.s. We define the stopping times

$$
\tau_{t}^{\mathrm{Q}}:=\inf \left\{s>0 \mid\left\langle\bar{L}^{\mathrm{Q}}\right\rangle_{s}>t\right\}
$$

for any $t \geq 0$ and the process $B^{\mathrm{Q}}=\left(B_{t}^{\mathrm{Q}}\right)_{t \geq 0}$ by

$$
B_{t}^{\mathrm{Q}}:=\bar{L}_{\tau_{t}^{\mathrm{Q}}}^{\mathrm{Q}} .
$$

Then the Dambis-Dubins-Schwarz theorem (see Theorem 3.4.6 of [3]) implies that $B^{\mathrm{Q}}$ is a Brownian motion. Set $\tau^{\mathrm{Q}}:=\tau_{c_{1} T}^{\mathrm{Q}} \wedge T$. By definition, $\tau^{\mathrm{Q}}$ is a stopping time with respect to $\mathbb{F}$ and values in $[0, T]$. Moreover, as $\bar{L}^{\mathrm{Q}}=L^{\mathrm{Q}}$ on $\llbracket 0, T \rrbracket$ and $\left\langle\bar{L}^{\mathrm{Q}}\right\rangle$ is continuous, we obtain

$$
G_{T}^{\mathrm{Q}}=\left\{\left\langle\bar{L}^{\mathrm{Q}}\right\rangle_{T}>c_{1} T\right\} \subseteq\left\{\tau_{c_{1} T}^{\mathrm{Q}}<T\right\}=\left\{\tau_{c_{1} T}^{\mathrm{Q}}=\tau^{\mathrm{Q}}<T\right\}
$$

We also note that, for any standard normal random variable $U$, we have the estimate

$$
\mathrm{P}[U>a b] \leq \frac{1}{\sqrt{2 \pi} a} \mathrm{e}^{-a^{2} b^{2} / 2}
$$

for any $a>0$ and $b \geq 1$. For the set $A_{T}^{\mathrm{Q}}:=\left\{Z_{\tau^{\mathrm{Q}}}^{\mathrm{Q}}>\mathrm{e}^{-\delta T}\right\} \in \mathcal{F}_{\tau} \mathrm{Q} \subseteq \mathcal{F}_{T},(2.10),(2.7)$, Lemma 2.4, (2.9), (2.11), and (2.8) then yield

$$
\begin{aligned}
\mathrm{P}\left[A_{T}^{\mathrm{Q}}\right] & =\mathrm{P}\left[\left\{Z_{\tau^{\mathrm{Q}}}^{\mathrm{Q}}>\mathrm{e}^{-\delta T}\right\} \cap G_{T}^{\mathrm{Q}}\right]+\mathrm{P}\left[\left\{Z_{\tau^{\mathrm{Q}}}^{\mathrm{Q}}>\mathrm{e}^{-\delta T}\right\} \cap\left(G_{T}^{\mathrm{Q}}\right)^{\mathrm{c}}\right] \\
& \leq \mathrm{P}\left[Z_{\tau_{c_{1} T}^{\mathrm{Q}}}^{\mathrm{Q}}>\mathrm{e}^{-\delta T}, \tau_{c_{1} T}^{\mathrm{Q}}=\tau^{\mathrm{Q}}<T\right]+\mathrm{P}\left[\left(G_{T}^{\mathrm{Q}}\right)^{\mathrm{c}}\right] \\
& =\mathrm{P}\left[L_{\tau_{c_{1} T}^{\mathrm{Q}}}^{\mathrm{Q}}-\frac{1}{2}\left\langle L^{\mathrm{Q}}\right\rangle_{\tau_{c_{1} T}^{\mathrm{Q}}}>-\delta T, \tau_{c_{1} T}^{\mathrm{Q}}=\tau^{\mathrm{Q}}<T\right]+\mathrm{P}\left[\left(G_{T}^{\mathrm{Q}}\right)^{\mathrm{c}}\right] \\
& =\mathrm{P}\left[\bar{L}_{\tau_{c_{1} T}^{\mathrm{Q}}}^{\mathrm{Q}}-\frac{1}{2}\left\langle\bar{L}^{\mathrm{Q}}\right\rangle_{\tau_{c_{1} T}^{\mathrm{Q}}}>-\delta T, \tau_{c_{1} T}^{\mathrm{Q}}=\tau^{\mathrm{Q}}<T\right]+\mathrm{P}\left[\left(G_{T}^{\mathrm{Q}}\right)^{\mathrm{c}}\right]
\end{aligned}
$$




$$
\begin{aligned}
& \leq \mathrm{P}\left[B_{c_{1} T}^{\mathrm{Q}}-\frac{1}{2} c_{1} T>-\delta T\right]+\mathrm{P}\left[\left(G_{T}^{\mathrm{Q}}\right)^{\mathrm{c}}\right] \\
& =\mathrm{P}\left[B_{1}^{\mathrm{Q}}>\frac{c_{1}-2 \delta}{2 \sqrt{c_{1}}} \sqrt{T}\right]+\mathrm{P}\left[\left(G_{T}^{\mathrm{Q}}\right)^{\mathrm{c}}\right] \\
& \leq \frac{2 \sqrt{c_{1}}}{\left(c_{1}-2 \delta\right) \sqrt{2 \pi}} \exp \left(-\frac{\left(c_{1}-2 \delta\right)^{2}}{8 c_{1}} T\right)+\exp \left(-\frac{c_{2}}{2} T\right) .
\end{aligned}
$$

Combining this with (2.5) and (2.6) gives

$$
\mathrm{P}\left[A_{T}^{\mathrm{Q}}\right] \leq \tilde{C} \mathrm{e}^{-\gamma_{1} T}<1 .
$$

Moreover, we deduce, from the definition of $A_{T}^{\mathrm{Q}}$ and as $\delta=\gamma_{2}$, that

$$
\mathrm{Q}\left[A_{T}^{\mathrm{Q}}\right]=1-\mathrm{Q}\left[\left(A_{T}^{\mathrm{Q}}\right)^{\mathrm{c}}\right]=1-\mathrm{E}\left[Z_{\tau}^{\mathrm{Q}} \mathbf{1}_{\left(A_{T}^{\mathrm{Q}}\right)^{\mathrm{c}}}\right] \geq 1-\mathrm{e}^{-\gamma_{2} T} .
$$

Thanks to the quantitative strengthening achieved in Proposition 2.1, we are now able to prove the announced result.

Theorem 2.1. Under Assumption 1.1, suppose that a market price of risk $\lambda$ for the price process $S$ satisfies a large deviations estimate. Then $S$ allows asymptotic exponential arbitrage with exponentially decaying failure probability.

Proof. By Proposition 2.1, there exist $1 \leq T_{0}<\infty$ and constants $\tilde{C}, \gamma_{1}, \gamma_{2}>0$ such that, for any $T \geq T_{0}$, we can find for any $\mathrm{Q} \in \mathbb{M}_{m}^{T, e}$ a set $A_{T}^{\mathrm{Q}} \in \mathcal{F}_{T}$ with

$$
\mathrm{P}\left[A_{T}^{\mathrm{Q}}\right] \leq \tilde{C} \mathrm{e}^{-\gamma_{1} T}=: \varepsilon_{1, T}<1 \quad \text { and } \quad \mathrm{Q}\left[A_{T}^{\mathrm{Q}}\right] \geq 1-\mathrm{e}^{-\gamma_{2} T}=: 1-\varepsilon_{2, T} .
$$

In particular, $\gamma_{1} T>\log \tilde{C}$. For any $T \geq T_{0}$, we define

$$
\alpha_{T}:=\frac{\log \tilde{C}-\gamma_{1} T}{-\gamma_{2} T}>0 .
$$

Thus, $\alpha_{T}$ converges increasingly to $\gamma_{1} / \gamma_{2}$ as $T \rightarrow \infty$, and we have

$$
\varepsilon_{1, T}=\varepsilon_{2, T}^{\alpha_{T}}
$$

We take $T_{0} \leq \tilde{T}<\infty$ and a constant $\gamma_{3}$ with $0<2 \gamma_{3}<\gamma_{2} / 2$ such that, for any $T \geq \tilde{T}$,

$$
\mathrm{e}^{\left(\gamma_{2} / 2-\gamma_{3}\right) T}-1 \geq \mathrm{e}^{\gamma_{3} T} \text { and } 2^{1+\gamma_{1} / \gamma_{2}} \sqrt{\tilde{C} \mathrm{e}^{-\gamma_{1} T}}<1 .
$$

Now fix any $T \geq \tilde{T}$, and set $\tilde{\varepsilon}_{1, T}:=2^{1+\gamma_{1} / \gamma_{2}} \sqrt{\varepsilon_{1, T}}<1$ and $\tilde{\varepsilon}_{2, T}:=\sqrt{\varepsilon_{2, T}}<1$. By construction, due to (2.12), we have

$$
\tilde{\varepsilon}_{1, T} \tilde{\varepsilon}_{2, T}^{\alpha_{T}}=2^{1+\gamma_{1} / \gamma_{2}} \sqrt{\varepsilon_{1, T}} \sqrt{\varepsilon_{2, T}^{\alpha_{T}}} \geq 2^{1+\alpha_{T}} \sqrt{\varepsilon_{1, T}} \sqrt{\varepsilon_{2, T}^{\alpha_{T}}}=2^{1+\alpha_{T}} \max \left(\varepsilon_{1, T}, \varepsilon_{2, T}^{\alpha_{T}}\right) .
$$

Therefore, it follows from Lemma 2.5 that $S$ admits $\left(\tilde{\varepsilon}_{1, T}, \tilde{\varepsilon}_{2, T}\right)$-arbitrage up to time $T$, which means that there is $\bar{X}_{T} \in \mathbb{K}^{T}$ such that

(a) $\bar{X}_{T} \geq-\mathrm{e}^{-\gamma_{2} T / 2}$, P-a.s.,

(b) $\mathrm{P}\left[\bar{X}_{T} \geq 1-\mathrm{e}^{-\gamma_{2} T / 2}\right] \geq 1-2^{1+\gamma_{1} / \gamma_{2}} \sqrt{\varepsilon_{1, T}}$. 
We set $X_{T}:=\mathrm{e}^{\left(\gamma_{2} / 2-\gamma_{3}\right) T} \bar{X}_{T} \in \mathbb{K}^{T}, \gamma_{4}:=\gamma_{1} / 2>0$, and $C:=2^{1+\gamma_{1} / \gamma_{2}} \sqrt{\tilde{C}}>0$. Owing to the definition of $X_{T}$ and (2.13), we obtain

$$
\begin{aligned}
\mathrm{P}\left[X_{T} \geq \mathrm{e}^{\gamma_{3} T}\right] & \geq \mathrm{P}\left[X_{T} \geq \mathrm{e}^{\left(\gamma_{2} / 2-\gamma_{3}\right) T}-1\right] \\
& \geq \mathrm{P}\left[X_{T} \geq \mathrm{e}^{\left(\gamma_{2} / 2-\gamma_{3}\right) T}-\mathrm{e}^{-\gamma_{3} T}\right] \\
& =\mathrm{P}\left[\bar{X}_{T} \geq 1-\mathrm{e}^{-\gamma_{2} T / 2}\right] .
\end{aligned}
$$

Thus, we conclude from the above properties of $\bar{X}_{T}$ and the definition of $\varepsilon_{1, T}$ that

(a) $X_{T} \geq-\mathrm{e}^{-\gamma_{3} T}$, P-a.s.,

(b) $\mathrm{P}\left[X_{T} \leq \mathrm{e}^{\gamma_{3} T}\right] \leq C \mathrm{e}^{-\gamma_{4} T}$,

which proves the assertion.

As a direct corollary, we can prove the conjecture in [1].

Corollary 2.1. Let $(\tilde{\Omega}, \tilde{\mathcal{F}}, \tilde{\mathbb{F}}, \tilde{\mathrm{P}})$ be a filtered probability space where the filtration $\tilde{\mathbb{F}}=\left(\tilde{\mathcal{F}}_{t}\right)_{t \geq 0}$ is the $\tilde{\mathrm{P}}$-augmentation of the raw filtration generated by an $\mathbb{R}^{N}$-valued Brownian motion $\tilde{\tilde{W}}$. Moreover, let $\tilde{S}$ be the diffusion process defined in (1.2). Suppose that the market price of risk function $\varphi(\cdot)$ satisfies a large deviations estimate with respect to $\tilde{S}$. Then $\tilde{S}$ allows asymptotic exponential arbitrage with exponentially decaying failure probability.

Proof. By our assumption (1.3) on the diffusion process in (1.2) and the choice of the filtration $\tilde{\mathbb{F}}$, the martingale representation theorem implies Assumption 1.1 for $\tilde{\mathrm{P}}$. Moreover, it is well known that, for every $T<\infty$ and any equivalent martingale measure $\tilde{Q}$ for $\left(\tilde{S}_{t}\right)_{0 \leq t \leq T}$, the density process $\tilde{Z}^{\tilde{Q}}$ with respect to $\left.\tilde{\mathrm{P}}\right|_{\tilde{F}_{T}}$ is of the form

$$
\tilde{Z}^{\tilde{\mathrm{Q}}}=\mathcal{E}\left(\int-\psi^{\tilde{\mathrm{Q}}} \mathrm{d} \tilde{W}\right)=: \mathcal{E}\left(\tilde{L}^{\tilde{\mathrm{Q}}}\right),
$$

where $\left(\psi_{t}^{\tilde{\mathrm{Q}}}\right)_{0 \leq t \leq T}$ is a predictable $\mathbb{R}^{N}$-valued process with $\psi_{t}^{\tilde{\mathrm{Q}}}-\varphi\left(\tilde{S}_{t}\right) \in \operatorname{ker}\left(\sigma\left(\tilde{S}_{t}\right)\right)$ for any $t \in[0, T]$. As a consequence, we have

$$
\left\langle\tilde{L}^{\tilde{\mathrm{Q}}}\right\rangle_{t}=\int_{0}^{t}\left\|\psi_{s}^{\tilde{\mathrm{Q}}}\right\|^{2} \mathrm{~d} s \geq \int_{0}^{t}\left\|\varphi\left(\tilde{S}_{s}\right)\right\|^{2} \mathrm{~d} s
$$

for any $t \in[0, T]$. For details, we refer the reader to Section 3 of [1]. Therefore, if we compare Definitions 1.2 and 1.3 and look at Lemma 2.2, we see that we get the result directly by using the same computations as in Proposition 2.1 and Theorem 2.1 , replacing $L^{\mathrm{Q}}$ by $\tilde{L}^{\tilde{\mathrm{Q}}}$ and $K$ by $\int\left\|\varphi\left(\tilde{S}_{S}\right)\right\|^{2} \mathrm{~d} s$.

Föllmer and Schachermayer [1] considered the diffusion process $\tilde{S}$ defined in (1.2) and introduced a quantitative form of long-term arbitrage. This is almost the same as asymptotic exponential arbitrage with exponentially decaying failure probability, with the difference that there is no relation between the tolerance level of failure and the necessary time to reach a level. The authors introduced the notion of having an average squared market price of risk above a threshold $c>0$, which is a growth condition on the mean-variance tradeoff process. They proved that if $\tilde{S}$ satisfies this then there exists the above kind of long-term arbitrage (see Theorem 1.4 of [1]). Furthermore, the authors wrote that one should expect to have asymptotic exponential arbitrage with exponentially decaying failure probability (in the sense of the present 
paper) under the stronger assumption that the market price of risk function $\varphi(\cdot)$ for $\tilde{S}$ satisfies a large deviations estimate. They even sketched an argument how one could try to prove this conjecture using a large deviations approach, but left the details and precise assumptions open. In Mbele Bidima and Rásonyi [4], the authors proved such a result in a discrete-time version of the model (1.2) by using a large deviations estimate for a martingale difference sequence (see Theorem 4 of [4]). The main contribution of the present paper is a rigorous proof based on a time-change argument instead of a large deviations approach. In addition to avoiding any extra assumptions, this has also allowed us to prove the result not only for diffusions, but for general continuous semimartingales (satisfying Assumption 1.1).

\section{Acknowledgements}

The authors wish to thank Martin Schweizer for his careful reading, remarks, and suggestions which have improved the presentation of this paper. Financial support from the National Centre of Competence in Research 'Financial Valuation and Risk Management' (NCCR FINRISK), Project D1 (Mathematical Methods in Financial Risk Management) is gratefully acknowledged. The NCCR FINRISK is a research instrument of the Swiss National Science Foundation.

\section{References}

[1] Föllmer, H. and Schachermayer, W. (2007). Asymptotic arbitrage and large deviations. Math. Finance Econom. 1, 213-249.

[2] Jacod, J. And Shiryaev, A. N. (2003). Limit Theorems for Stochastic Processes (Fundamental Principles Math. Sci. 288), 2nd edn. Springer, Berlin.

[3] Karatzas, I. And Shreve, S. E. (2000). Brownian Motion and Stochastic Calculus, 2nd edn. Springer, Berlin.

[4] Mbele Bidima, M. L. D. ANd RÁsonyi, M. (2012). On long-term arbitrage opportunities in Markovian models of financial markets. Ann. Operat. Res. 200, 131-146.

[5] Schweizer, M. (1995). On the minimal martingale measure and the Föllmer-Schweizer decomposition. Stoch. Anal. Appl. 13, 573-599. 